\title{
Biliary intraductal papillary mucinous neoplasm visualized intralesionally through a fistula with the duodenal bulb
}

A 53-year-old man was admitted to our hospital with abdominal pain. He had undergone surgery for a pancreatic pseudocyst related to chronic alcoholic pancreatitis 7 years previously at another hospital. Magnetic resonance imaging (MRI) 3 years later had shown a cystic lesion, $4 \mathrm{~cm}$ in diameter, at the hepatic hilum, with two small mural nodules. The patient was then lost to follow-up for 3 years.

Following admission to our hospital, MRI showed a $6 \times 6.5-\mathrm{cm}$, inhomogeneous solid lesion between the liver, pancreas, and duodenum, with compression of the duodenal bulb ( Fig. 1 a) and invasion of the left biliary duct, common hepatic duct ( $\bullet$ Fig. 1 b), hepatic artery, and portal vein. Gastroscopy showed the presence of a compression on the anterior wall of the duodenal bulb. The compression had two fistulous orifices with villous tissue at the edges, which were exuding whitish mucus ( Fig.2a). A diagnostic gastroscope was inserted through one of the fistulous orifices revealing that the internal structure of the neoplastic lesion that was forming a fistula to the duodenal bulb was a multiloculated cavity covered with a whitish, villous tissue ( Fig. $\mathbf{2 b - d}$; - Video 1). A biopsy of the villous tissue at the edge of the fistula revealed fragments of epithelial neoplasia with papillary structures and focal adenocarcinomatous transformation ( $\mathbf{F i g} \cdot \mathbf{3 a}$ ).

The patient underwent surgery, and a neoplastic lesion that was infiltrating the biliary tract and the vascular structure at the hepatic hilum, as well as fistulizing to the duodenal bulb, was seen. The pancreas was normal. The surgeon decided to perform a gastrodigiunal anastomosis. Histology of the surgical sample showed an adenocarcinoma, consistent with a biliary intraductal papillary mucinous neoplasm (IPMN-B), the papillary struc-

Video 1

Endoscopic examination of the internal structure of the neoplastic lesion that was forming a fistula to the duodenal bulb, showing a multiloculated cavity covered by a whitish, villous tissue.
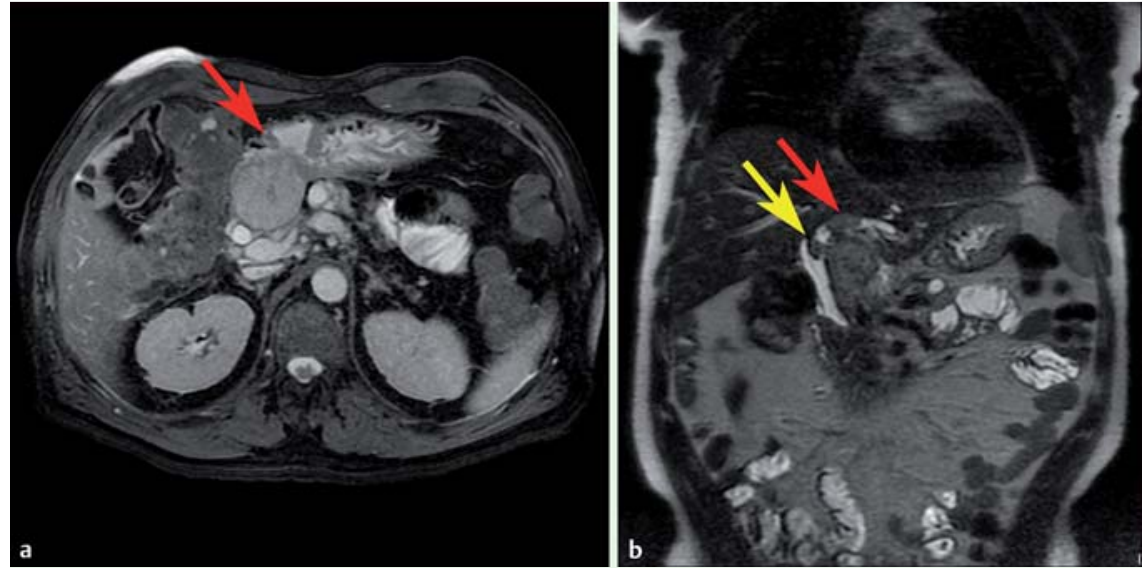

Fig. 1 Magnetic resonance imaging (MRI) from a 53-year-old man who presented with abdominal pain showing: $\mathbf{a}$ an inhomogeneous solid lesion between the liver, pancreas, and duodenum, which is compressing the duodenal bulb (red arrow); $\mathbf{b}$ evidence of invasion of the left biliary duct (red arrow) and the common hepatic duct (yellow arrow).
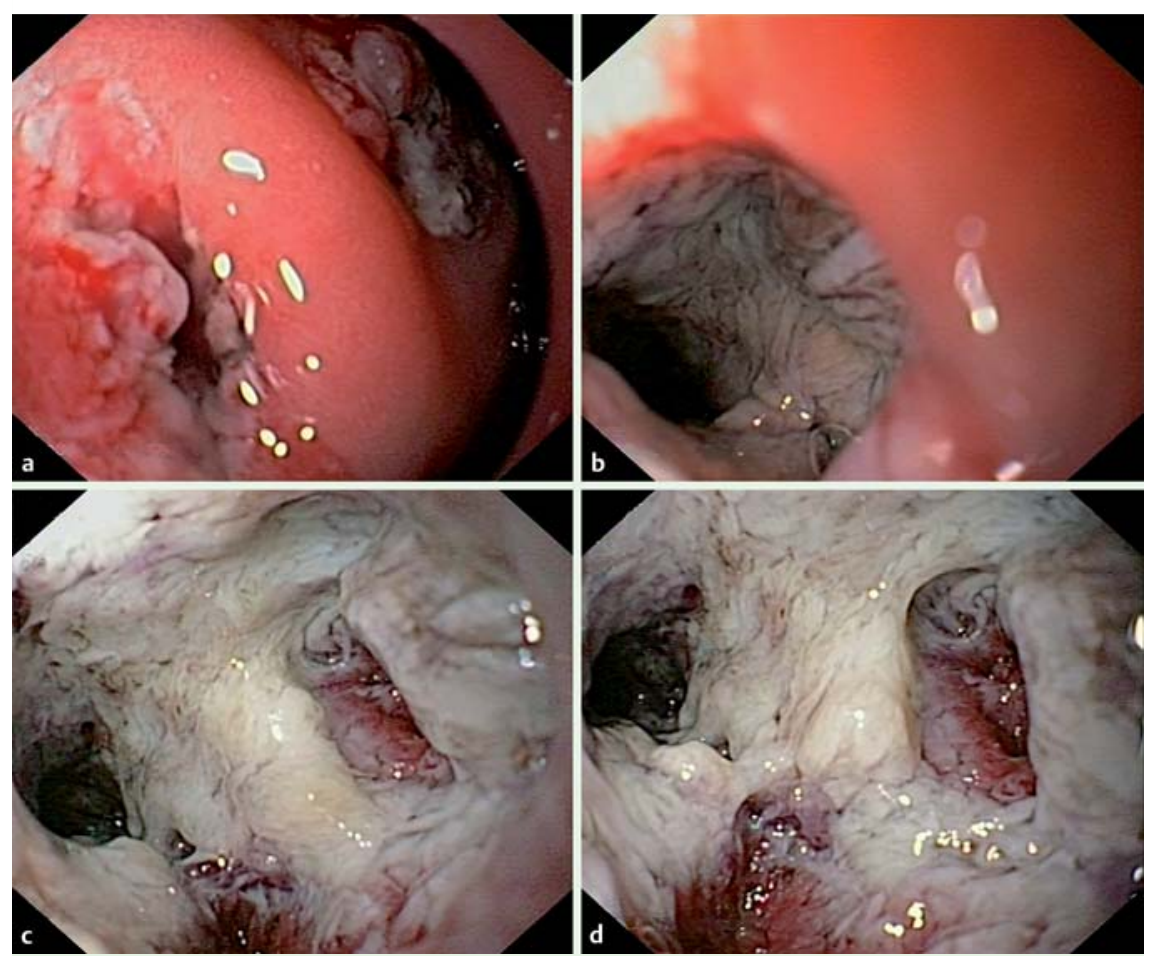

Fig. 2 Endoscopic views of: a the duodenal bulb compression with two fistulous orifices edged by villous tissue; $\mathbf{b}-\mathbf{d}$ the neoplastic lesion that was forming a fistula to the duodenal bulb, showing a multiloculated cavity covered by a whitish, villous tissue. 


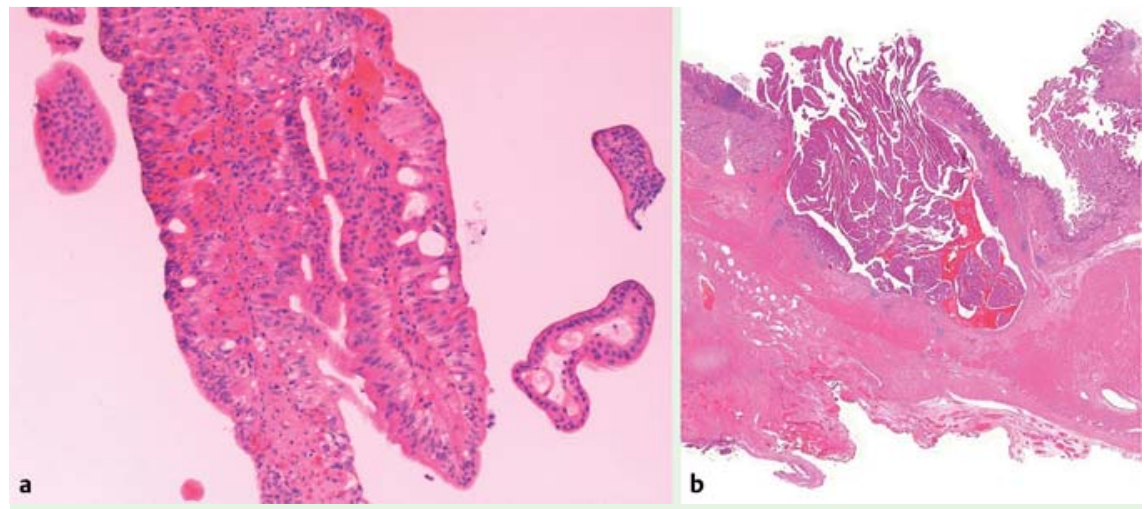

Fig.3 Histological appearances of the neoplastic lesion from: a endoscopic biopsy fragments showing epithelial neoplasia with papillary structures and focal adenocarcinomatous transformation; $\mathbf{b}$ the surgical resection showing adenocarcinoma, compatible with biliary intraductal papillary mucinous neoplasm (IPMN-B), with papillary structures that are fistulizing to the duodenal bulb.

tures of which were fistulizing to the duodenal bulb $(\bullet$ Fig.3b).

IPMN-B is a type of bile duct tumor with certain unique features, which include intraductal papillary growth and mucin production. Recent studies have revealed pathological similarities between the IPMN-B and pancreatic IPMN (IPMN-P), and some even consider the two neoplasms to be counterparts [1,2]. There are several reports of IPMN-P fistulizing to surrounding organs [3-5]; however, to the best of our knowledge, this is the first report of an IPMN-B forming a fistula with the duodenal bulb. This occurrence appears to confirm a similarity in behavior between IPMN-B and IPMN-P.
Endoscopy_UCTN_Code_CCL_1AB_2AZ_3AB

Competing interests: None

\section{Barresi, I. Tarantino, A. Granata, G. Curcio, R. Gentile, R. Liotta, G. Marrone, M. Traina}

Gastroenterology and Endoscopy Service, IsMeTT/UPMC, Palermo, Italy

\section{References}

$1 \mathrm{Kim} \mathrm{HJ}$, Kim MH, Lee SK et al. Mucin hypersecreting bile duct tumor characterized by a striking homology with an intraductal papillary mucinous tumor (IPMT) of the pancreas. Endoscopy 2000; 32: 389-393

2 Zen $Y$, Fujii T, Itatsu $\mathrm{K}$ et al. Biliary papillary tumors share pathological features with intraductal papillary mucinous neoplasm of the pancreas. Hepatology 2006; 44: $1333-$ 1343

3 Jasset F, Delvaux M, Dumitriu D et al. Benign intraductal papillary-mucinous neoplasm of the pancreas with spontaneous associated pancreaticogastric and pancreaticoduodenal fistula. Digestion 2010; 82: $42-46$

4 Jung IS, Shim CS, Cheon YK et al. Invasive intraductal papillary mucinous tumor of the pancreas with simultaneous invasion of the stomach and duodenum. Endoscopy 2004; 36: $186-189$

5 Barresi L, Tarantino I, Curcio G et al. Endoscopic ultrasound (EUS) aspect of duodenal bulb invasion by multifocal malignant pancreatic intraductal papillary neoplasm (IPMN). Endoscopy 2011; 43 (Suppl. 2): E270 - E271

\section{Bibliography}

DOI http://dx.doi.org/

10.1055/s-0031-1291653

Endoscopy 2012; 44: E84-E85

(c) Georg Thieme Verlag KG

Stuttgart · New York

ISSN 0013-726X

\section{Corresponding author \\ Luca Barresi, MD}

Gastroenterology and Endoscopy Service IsMeTT, UPMC

Via Tricomi 1

Palermo 90100

Italy

Fax: +39-091-2192400

Ibarresi@ismett.edu 